\title{
Técnica de infiltración perineural en heridas complejas (PIT)
}

\author{
Perineural infiltration technique (PIT) in complex wounds
}

\author{
Héctor Tito Leoni' ${ }^{1}$ Francisco Olivero Vila르. Andrés Amoroso ${ }^{3}$, Ana María Lupia ${ }^{4}$, Marcela Botargues ${ }^{5}$
}

\section{RESUMEN}

El dolor asociado a heridas complejas se erige como una gran barrera para la resolución de estas, debido a la complejidad de abordaje y a la imposibilidad de establecer un patrón de algoritmo por diversos factores y su carácter heterogéneo, así como por la presencia de manifestaciones múltiples asociadas.

Este dolor provoca al paciente un daño no solo físico sino también psicosocial, porque la escasa respuesta a los tratamientos habituales, y si bien es sensible la medicación con opiáceos, los resultados son insuficientes a largo plazo en un significativo número de pacientes, en quienes provoca además efectos no deseables.

Se establece en el presente trabajo la administración subcutánea de dextrosa 5\% a través de una técnica de infiltración perineural para el tratamiento de la inflamación neurogénica responsable del mencionado padecimiento, en las terminaciones libres de los nervios sensitivos cutáneos.

Para ello se decidió realizar un estudio descriptivo multicéntrico a partir dejunio del 2016 hasta mayo del 2017 en 60 pacientes que presentaban dolor crónico en ulceras de miembro inferior de diferente etiologí con antecedente de medicación analgésica previa con resultado parcial (no controlado) y que tuviera evolución de su dolor entre 3 meses y 4 años.

Palabras claves: dolor crónico, infiltración perineural subcutánea, heridas complejas.

\begin{abstract}
The pain associated with complex wounds is established as a great barrier for the irresolution, due to the complexity of the approach and the impossibility of establishing an algorithm pattern due to various factors and their heterogeneous nature, as well as the presence of associated multiple manifestations.

This pain causes not only physical but psychosocial damage to the patient, because of the poor response to the usual treatments, and although it is sensitive to medication with opiates, the results are insufficient in the long-term in a significant number of patients, in whom it also causes undesirable effects.

The present work establishes the subcutaneous administration of 5\% dextrose through a perineural infiltration technique for the treatment of the neurogenic inflammation responsible for the afore mentioned condition, in the free terminations of the cutaneous sensory nerves.

For this, it was decided to carry out a multicenter descriptive study from June 2016 to May 2017 in 60 patients who presented chronic pain in lower limb ulcers of different etiology with a history of previous analgesic medication with partial (uncontrolled) outcome and had evolution of your pain between 3 months and 4 years.
\end{abstract}

Key words: chronic pain, subcutaneous perineural infiltration, complex wounds.

\section{INTRODUCCIÓN}

Si definimos el dolor como una experiencia sensorial y emocional desagradable con daño tisular real, potencial o descrito en términos de ese daño (OMS), cuando ese dolor es de corta duración o tiene un propósi-

1. Médico Especialista Universitario en Cirugía Plástica, Médico de Planta del servicio de Cirugía Plástica del Instituto de Rehabilitación Psicofísica "IREP" y jefe de la Clínica de Heridas, Ciudad Autónoma deBuenos Aires, Argentina

2. Médico Especialista Universitario en Cirugía Plástica, jefe de servicio de Cirugía Plástica del Instituto de Rehabilitación Psicofísica "IREP", Profesor titular de la carrera de Cirugía Plástica de la Universidad del Salvador

3. Médico Especialista Universitario en Cirugía Plástica, Universidad del Salvador

4. Médica Especialista en Medicina Interna, Médica Asociada Hospital Italiano, Buenos Aires,Consultorio de Dolor, Proloterapia y Terapia de Inyecciones Perineurales. Secretaria de la LAOM (Asociación Latinoamericana de Medicina Ortopédica Regenerativa)

5. Médica de Familia del Hospital Italiano de Buenos Aires

$\bowtie$ Correspondencia: Héctor Tito Leoni. hectorleoni2000@gmail.com

Los autores no declaran conflictos de intereses

Trabajo presentado en el $47^{\circ}$ Congreso Argentino de Cirugía Plástica, Estética y Reparadora, La Plata,Pcia. de Buenos Aires,mayo 2017

Recibido: 04/06/2019 / Aceptado: 12/10/2019 to como la curación, el paciente tiende a aceptarlo y tolerarlo. Este no es el caso del dolor crónico (DC), que compromete al sistema somatosensorial, provocando un impacto en la calidad de vida del paciente ${ }^{1,2}$ (Tabla 1).

El DC asociado a heridas complejas se erige como una gran barrera para su resolución, no solo por sus características y complejidad de abordaje, sino porque además los profesionales de la salud focalizan su atención en el manejo de la lesión y su etiología por encima de tratamiento de aquel ${ }^{3}$.

Esta prioridad no es vista de igual manera por el paciente, quien padece un cuadro desgastante con evidentes signos de compromiso general y repercusiones psicosociales y de relación individual, conductual y con una serie característica de manifestaciones que van más allá de una herida, alterando su evolución y distorsionando los resultados de los diferentes tratamientos. Las heridas aún con diversas etiologías presentan dolor de características mixtas -somático y neuropáticoporque las múltiples causas de su origen pueden asociarse o superponerse.

Es frecuente que los pacientes con úlceras refieran entre sus síntomas: pinchazos, ardor, picor, calor con características de dolor neuropático (DN) (Tabla 2), y este dolor por definición actual de la IASP (Internatio- 
TABLA 1. Dolor agudo y dolor crónico.

\begin{tabular}{|c|c|}
\hline Agudo & Crónico \\
\hline Inicia por daño tisular & Inicio como agudo. >8 semanas \\
\hline $\begin{array}{l}\text { Desaparece con la remisión de la } \\
\text { herida }\end{array}$ & $\begin{array}{l}\text { Destructivo física, psicológica y so- } \\
\text { cialmente (carece de valor biológico) }\end{array}$ \\
\hline Proporcional a la lesión que lo causa & Mayor duración que la esperada \\
\hline Responde a tratamientos & $\begin{array}{l}\text { El grado de doolor no refleja el gra- } \\
\text { do de daño tisular (alodinia o hipe- } \\
\text { ralgesia) }\end{array}$ \\
\hline Respuesta escasa a opiáceos & No relación con magnitud de lesión \\
\hline \multirow[t]{5}{*}{ Síntoma (reflejo sensorial protector) } & No responde a ttos del daño físico \\
\hline & Responde a opiáceos \\
\hline & Asociado a depresión, ansiedad, etc. \\
\hline & $\begin{array}{l}\text { Respuesta orgánica: anorexia, in- } \\
\text { somnio, disminuye la libido, agota- } \\
\text { miento físico }\end{array}$ \\
\hline & Es una ENFERMEDAD. \\
\hline
\end{tabular}

nal Association for the Study of Pain) corresponde a "lesión o enfermedad del sistema somatosensorial”, siendo las posibles causas de estas lesiones el origen isquémico, infiltrativo, inflamatorio, por destrucción o atrapamiento del nervio, causas que confluyen en el origen de las úlceras ${ }^{4}$.

Los diferentes factores y diversas manifestaciones que acompañan al DC hacen dificultoso establecer un algoritmo de tratamiento.

$\mathrm{Si}$ bien existen alternativas fisioterapéuticas y farmacológicas para tratar el dolor en estos pacientes, no resultan suficientes ya que solo la mitad de los que padecen DC presentan respuesta al tratamiento, y a veces solo un alivio parcial 5 . Debido a esto se plantean nuevas alternativas para el tratamiento del dolor, que complementen las existentes.

En la búsqueda de trabajos relacionados con el manejo actual del dolor se hace referencia a los mínimos efectos de los opioides a largo plazo, pudiendo causar: dependencia, tolerancia, somnolencia, deterioro del juicio, de la memoria, de la atención (concentración), constipación, por lo que la IASP recomienda precaución para su prescripción en el dolor crónico y pone un mayor énfasis en el control no opioide del mismo ${ }^{3}$.

Diversos métodos no opioides para el manejo del dolor se utilizan con diferentes grados de efectividad. Entre las alternativas del tratamiento local en patologías que involucran $\mathrm{DN}$, uno de los agentes más utilizados es el parche de lidocaína en una dosis de 1,8-5\%, aprobado por la FDA (Food and Drug Administration) para la neuralgia posherpética y recomendado para terapia del dolor periférico con escasos efectos secundarios y ausencia de efectos a nivel del sistema nervioso central. Además, en relación con las úlceras venosas de piernas y el dolor incidental (por desbridamiento), se evaluó la efectividad de utilizar analgesia local (MEAL: mezcla eutéctica de anestésicos locales, prilocaína asociada a lidocaína), comprobándose el alivio efectivo del dolor basado en una revisión sistemática del Grupo de Heridas Cochrane $2002^{6}$.

Otro fármaco utilizado, llamado capsaicina ${ }^{7}$, es una sustancia alcaloide del pimiento Capsicum. Es el ingre-
TABLA 2. Dolor neuropático

\begin{tabular}{|ll|}
\hline - & Dolor ANORMAL. No nociceptivo \\
\hline - & $\begin{array}{l}\text { Contico } \\
\text { sias (hormo (quemazón), fulgurante (descarga eléctrica), diseste- }\end{array}$ \\
\hline - & Depresión, insomnio, ansiedad, anorexia \\
\hline - & Dependencia a sustancias psicoactivas \\
\hline - & Autta de adherencia a tratamintos \\
\hline - & Impacto afectivo y sociolaboral negativo \\
\hline
\end{tabular}

diente picante de estos pimientos y actúa en receptores vaniloides de los nervi nervorum llamados TRVP1; la capsaicina es utilizada como parche al $8 \%$ como segunda línea de tratamiento del dolor de la neuralgia posherpética y del DN, presentando como efectos secundarios locales reacciones e importantes molestias en la aplicación inicial.

Se utiliza también en la actualidad morfina en forma tópica en hidrogel o líquida (combinada con lidocaína por sus diferentes tipos de inicio y duración), en base a algunos trabajos publicados que lo avalan, teniendo en cuenta que no se han podido comprobar efectos sistémicos, y basado en la cantidad de receptores opioides que hay en la superficie ${ }^{8,9}$. Además, existen numerosos trabajos que hacen referencia a la utilización de dextrosa en forma infiltrativa como analgésico eficaz.

En los últimos 50 años se han publicado numerosos estudios en los cuales se describe la utilización de dextrosa para el tratamiento del dolor musculoesquelético crónico mediante la técnica conocida como proloterapia ${ }^{10}$.

La proloterapia se puede definir como un tratamiento médico complementario, que consiste en la inyección de un agente "irritante" que busca generar un proceso inflamatorio de un tejido con el objetivo de aliviar el dolor, mejorar la función, estimular la reparación y curación de dichos tejidos. Esta técnica fue descrita en el siglo V a. C. por Hipócrates (460-370 a. C.) para el tratamiento de articulaciones "inestables", aplicando calor intenso sobre sus ligamentos en busca de obtener una respuesta inicial clínicamente inflamatoria, dando lugar a la subsecuente reparación ${ }^{11}$.

A mediados del siglo XIX, el científico médico Claude Bernard (1813-1878) introdujo el concepto de homeostasis e hizo referencia a que pequeñas fibras nerviosas sensibles a capsaicina se encargan de su modulación y equilibrio ${ }^{12}$.

En la década de 1940, el Dr. George S. Hackett usó diversas técnicas de inyección, sustancias inyectables e infiltración para el tratamiento del dolor de origen musculoesquelético a través de la inducción de la proliferación de nuevas células capaces de producir tejido fibroso, el cual reforzará y estabilizará la articulación eliminando permanentemente la discapacidad. Acuñó el término proloterapia (prolo, abreviación para la terapia proliferativa) para describir la técnica ${ }^{13}$. 


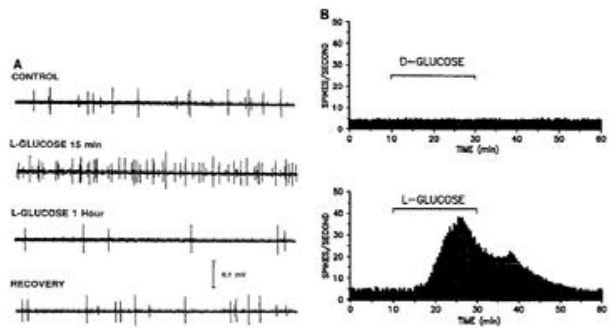

Figura 1. Hipoglucemia de fibras C. Maclver MB, Tanelian DL. Activation of $C$ fibers by metabolic perturbations associated with tourniquet ischemia. Anesthesiology 1992;76(4):617-23.

El efecto beneficioso de la dextrosa en altas concentraciones $(25$ o $12,5 \%)$ sobre el dolor ha sido documentado en pacientes con dolor osteomioarticular (tendinosis del tendón de Aquiles, lesiones del manguito rotador, lumbalgia crónica, entre otros).

Dentro de las técnicas descritas en proloterapia se encuentra la terapia de inyecciones perineurales del Dr. J. Lyftogt denominada PIT (perineural injection therapy) también conocida como neuroproloterapia ${ }^{14}$.

Dicha técnica también utiliza la dextrosa pero, a diferencia de las anteriores técnicas descritas, utiliza una concentración significativamente menor que aplica exclusivamente a nivel subcutáneo en la proximidad de las terminaciones nerviosas sensoriales libres y de los nervios sensitivos periféricos afectados y responsables de la inervación de la zona injuriada.

El mecanismo de acción es todavía desconocido, y hay trabajos en desarrollo aunque la hipótesis a considerar hasta la fecha es que dicha infiltración ejerce un efecto supresor de la inflamación neurogénica, actuando directamente sobre los receptores TRPV1 (receptor de potencial transitorio valinoide) que asientan en terminaciones asinápticas nerviosas sensoriales cutáneas de los nervi nervorum de las fibras $\mathrm{C}$ amielínicas, favoreciendo el ingreso de la glucosa al espacio intracelular. Estos receptores, conocidos antiguamente como receptores de capsaicina porque eran sensibles al componente picante del chile, son sensibles además a modificaciones de la temperatura (por encima de $\operatorname{los} 45^{\circ} \mathrm{C}$ ), estado de acidez ( $\mathrm{pH}$ bajo), a variaciones de la osmolaridad, a la lidocaína, a la presión capilar y a los niveles deficitarios de glucosa intracelular (esta característica multifacética les da el nombre de receptores polimodales).

Estos receptores, que responden ante cualquier tipo de injuria que modifique el equilibio homeostático, asientan como ya explicamos en las fibras de conducción lenta tipo C. Estas son las responsables de la transmisión de impulsos originados por la injuria tisular. Además, se postula que los nervi nervorum puede extenderse más alla del perineuro e involucrar al tejido circundante, guardando una estrecha relación con el trofismo tisular ${ }^{15}$.

Resumiendo: dichas terminaciones libres son sensibles a la deprivación de oxígeno, a la disminución de

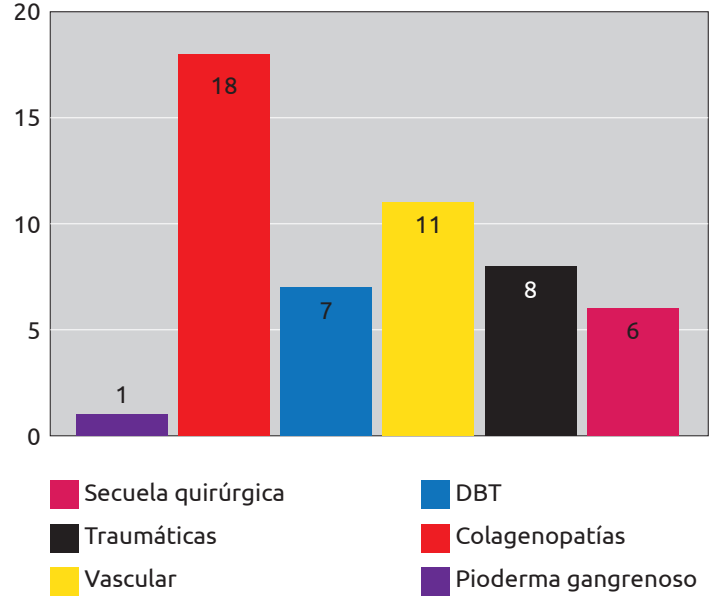

Figura 2.

glucosa intraneuronal (neuroglucopenia, Figura 1) y a un estado de acidosis, lo cual produce una crisis energética en el nervio. Este mecanismo propuesto se denomina OGD (deprivación de oxígeno y glu$\cos a)$.

Al alterarse la homeostasis en el entorno de las fibras C, los TRVP1 reaccionan con inflamación neurogénica pasando estos receptores de un estado de sleeping a un estado awake, generando dolor. El administrar glucosa iniciaría la recuperación de la homeostasis del nervio dañado al desactivar la inflamación neurogénica, volviendo a los receptores a un estado sleeping y desensibilizando el área comprometida. Se puede comprobar un alivio inmediato del dolor estimado en un $50 \%$ o más ${ }^{16}$.

La dextrosa al 5\% actuaría bloqueando el receptor TRPV1, disminuyendo la liberación de neuropéptidos proinflamatorios y, como consecuencia de ello, la inflamación neurogénica, restableciendo el trofismo y la homeostasis tisular. De esta manera, además, colaboraría en el proceso de cicatrización y reparación de los tejidos dañados.

Teniendo en cuenta el beneficio en diferentes afecciones musculoesqueléticas con DC, y debido a la insuficiente respuesta en muchos casos de los tratamientos establecidos en forma habitual, y como modificación y variante de la técnica descrita por el Dr. Lyftogt - PIT $-{ }^{17}$ que utiliza para el dolor osteomioarticular y otros usos en desarrollo, se decide la aplicación de este procedimiento para el tratamiento en úlceras crónicas de miembro inferior.

Dicha técnica establece la infiltración subcutánea de dextrosa al 5\% actuando a nivel de las terminaciones libres de los nervios cutáneos que inervan la zona injuriada y en forma periférica y circunferencial a la herida, pudiendo incrementarse el efecto analgésico extendiendo esta infiltración en forma proximal hacia las zonas de emergencia de los nervios (a través de la aponeurosis o fascia) que se detectan por palpación como puntos muy sensibles por irritación crónica (CCI) y 


\section{Respuesta a la cuarta semana}

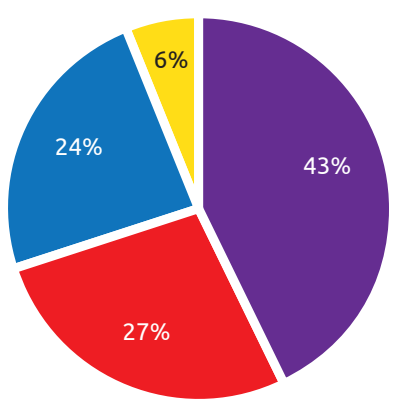

Sin respuesta

Leve a moderada respuesta
Aceptable respuesta

Eliminación de dolor

Figura 3.

que luego se dirigen según los mapas anatómicos hacia las zonas afectadas con heridas estando en conexión con ellas.

Estos nervios superficiales son los que transmiten la sensibilidad cutánea a la zona de la herida.

Se reconoce que entre las funciones del sistema somatosensorial y su rol en la propiocepción, el tacto fino, el dolor y la temperatura ${ }^{18}$, el funcionamiento interoceptivo está relacionado con el medio interno y está ligado a los niveles de glucosa, $\mathrm{pH}$ y oxígeno. Además de mantener el equilibrio de la homeostasis tisular, tiene como funciones el trofismo tisular (la Ley de Hilton indica que los nervios que cubren la piel están unidos con los nervios profundos que inervan las articulaciones, ligamentos y tendones en su camino hacia la médula espinal) con mecanismo de autorreparación de los tejidos, por lo que, ante la presencia de un dolor prolongado, también se ven comprometidos los mecanismos de cicatrización ${ }^{19}$. La administración de la técnica de infiltración perineural, en este caso, ayudaría a la reparación del tejido injuriado como efecto beneficioso complementario.

La técnica de infiltración perineural subcutánea restablecería a las pequeñas fibras asinápticas el nivel de acidez y glucosa normales, revirtiendo la inflamación neurogénica y disminuyendo el dolor (efecto desensibilizante), así como la homeostasis tisular (estado de equilibrio del tejido) al bloquear al receptor TRPV1. Además, actuaría en la regeneración tisular, teniendo en cuenta, como fue dicho anteriormente, que el tracto nervioso que inerva una articulación también inerva músculo, ligamentos, piel y tejidos circundantes ${ }^{18,19}$ Al plantearnos este trabajo, no existen en la actualidad publicaciones que refieran el uso de la técnica de infiltración perineural de dextrosa subcutánea para el tratamiento del DC relacionado con heridas complejas de miembros inferiores.

\section{OBJETIVOS}

- Demostrar efectividad de la infiltración perineural

Respuesta a los 3 meses

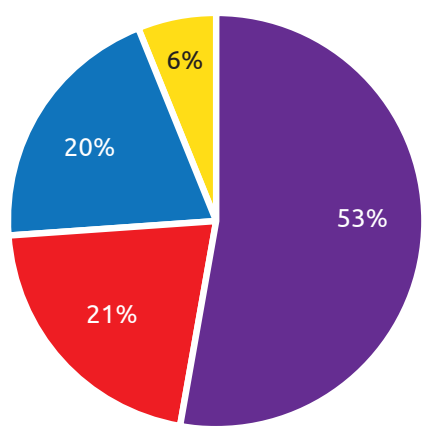

Sin respuesta

Aceptable respuesta

Leve a moderada respuesta

Eliminación de dolor

Figura 4.

subcutánea de dextrosa para el tratamiento de DC en lesiones ulcerosas de miembro inferior.

- Señalar el beneficio de la infiltración perineural subcutánea de dextrosa en el proceso de cicatrización.

- Aportar una herramienta efectiva, de bajo costo, fácil acceso, nunca antes utilizada para el tratamiento de heridas crónicas en miembro inferior.

\section{MATERIAL Y MÉTODO}

Se realizó un estudio descriptivo multicéntrico a partir de junio 2016 hasta mayo 2017 en 60 pacientes que presentaban DC en úlceras crónicas de miembro inferior de diferente etiología, con antecedente de medicación analgésica previa con resultado parcial y con tiempo de evolución de su dolor entre 3 meses y 4 años. Se realizó un cuestionario (DN4) para evaluar la prevalencia del dolor neuropático.

\section{CRITERIOS DE INCLUSIÓN}

- Paciente mayor de edad con una o más heridas en miembros inferiores de diversa etiología, con antecedentes de terapia analgésica insuficiente.

- DC asociado (EVA superior a 4).

- Herida con dificultad en el proceso de cicatrización.

- Pacientes que acepten las condiciones propuestas a través del protocolo, incluyendo consentimiento informado, periodicidad de procedimiento y registro fotográfico.

\section{CRITERIOS DE EXCLUSIÓN}

- Alodinia refractaria.

- Herida infectada.

- Ansiedad anticipatoria extrema no tratada.

- Intolerancia al procedimiento.

- Falta de compromiso e incumplimiento.

- Dolor de origen arterial no revascularizado y renuente. 


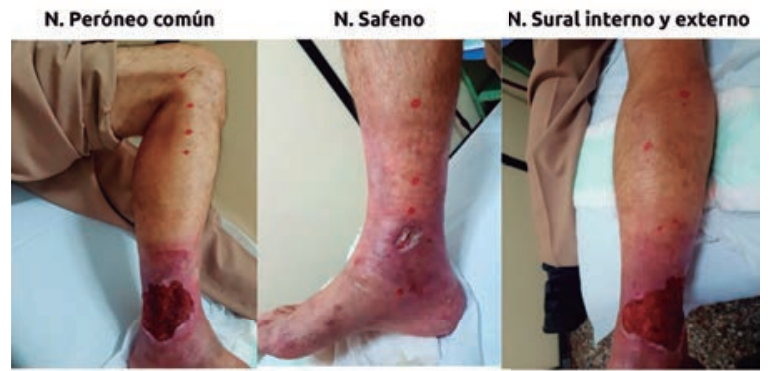

Puntos de Injuria Constrictiva Crónica (CCI)

Figura 5.

- Embarazo.

- Pacientes en mal estado general con evidente incapacidad de presentar un proceso adecuado de cicatrización.

Para llevar a cabo el siguiente trabajo, se siguió un método estándar de procedimiento, utilizando la técnica de infiltración subcutánea perineural de una solución de dextrosa al $5 \%$.

Este efecto inicial tiene una duración promedio de entre 2 y 4 días después de la primera infiltración, comenzando su efecto en 4-5 minutos.

Atento a la característica comunicacional del dolor, se utilizó para su valoración la escala analógica visual (EVA), a través de la cual el paciente refiere la magnitud álgica en una escala desde 0 (ausencia de dolor) hasta 10 (peor dolor padecido/imaginable). La evaluación se realizó cada 7 días y se utilizó para el seguimiento una adaptación de la Tabla de Recuperación de Proloterapia (TRP) ${ }^{20}$

Además, para profundizar la recolección de datos, se agregó un anexo que evalúa la calidad de vida y las limitaciones en actividades esenciales (caminar, vestirse, curación de herida, conciliación de sueño, trabajo) (Tabla 3). Se utilizó un agregado relacionado con la evolución del trofismo tisular que valora los parámetros de cierre de herida, lecho, bordes, piel perilesional, quedando el tamaño original registrado al comienzo de la TRP.

\section{DESCRIPCIÓN DE LA TÉCNICA (Figura 6)}

Los elementos a utilizar:

- Solución de dextrosa 5\% (1 sachet 500 cc, refrigerado a $4-5^{\circ} \mathrm{C}$ ).

- Jeringa de $1-5 \mathrm{ml}$.

- Agujas 25 G y $30 \mathrm{G}$.

- Antiséptico local tópico.

\section{PROTOCOLO}

- Marcación perilesional y puntos dolorosos (lesión constrictiva crónica).

- Antisepsia (yodo povidona diluida).

- Infiltración: En forma subcutánea con ángulo de $45^{\circ}$.

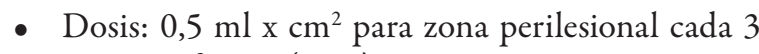
$\mathrm{cm}$ y periféricos (CCI).

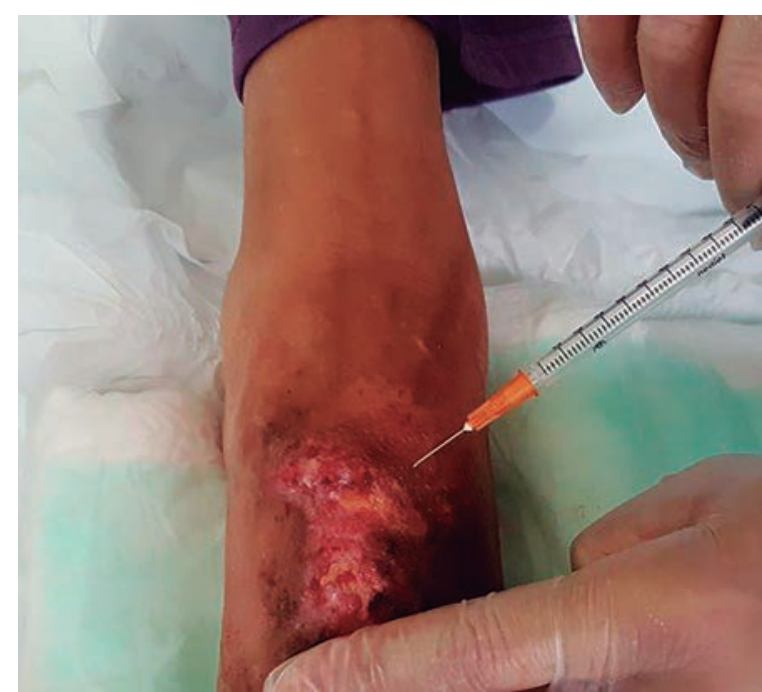

Figura 6.

- Medición del EVA a los 15 minutos.

- Curación de la herida con gel o solución de polihexametileno biguanida (PHMB) y gasas o loción de propóleos.

- Control de glucemia: glucotest pre- y posinfiltración alejada (30 minutos).

- Se reiteran como mínimo en 3 oportunidades más con un intervalo de 7 días, pudiendo extenderse hasta 8 o más sesiones de acuerdo a la evolución.

\section{RESULTADOS}

De los 60 pacientes incluidos, se registraron 51 debido a una tasa de deserción del 15\% (9 pacientes). La mayoría tenía una edad superior a 55 años y presentaban úlceras con dolor intolerable, promedio EVA 7, a pesar de la medicación habitual. El 45,1\% de los pacientes (23), de acuerdo al DN4, padecía dolor neuropático.

Luego de la infiltración subcutánea perineural, un alto porcentaje experimentó una mejoría significativa $(>80 \%)$ y alivio de la sintomatología inmediata del dolor ( 5 minutos), incrementándose la mejoría sustancialmente a partir de las 24 a 48 horas de la infiltración, en las cuales varios pacientes manifiestan supresión del síntoma.

La duración del efecto analgésico fue de aproximadamente 3,5 días. La reaparición álgica fue referida como de aparición lenta, sin alcanzar en la mayoría de los casos el nivel EVA inicial.

Al cabo de la cuarta sesión, un número importante de pacientes $(43,1 \%, 22$ pacientes) no presentaba dolor; $27,5 \%$ (14) lo presentaba de forma esporádica, leve y de corta duración; 23,52\% (12 pacientes), con disminución leve de dolor entre 1 y 2 puntos de la escala EVA; y 5,9\% (3 pacientes) sin respuesta definitiva a pesar de haber mejorado al comienzo del tratamiento.

Cabe consignar que los pacientes con DC de origen arterial fueron los que necesitaron mayor tiempo para 
TABLA 3. Tabla de recuperación (prolotherapy recovergram) Ejemplo de un caso $^{20}$.

\begin{tabular}{|l|l|}
\hline Nombre: $N N$ & Dirección: $x x x$ \\
\hline Hc: $x x x$ & Diagnóstico: úlcera vasculítica en la pierna \\
\hline Edad: 61 años & Medicación analgésica: $x x x$ \\
\hline Teléfono: $x x x$ & Fecha: dd/mm/aa \\
\hline
\end{tabular}

\begin{tabular}{|l|l|l|l|l|l|l|l|l|l|l|l|l|}
\hline \multicolumn{1}{|l|}{ EVA: 0 (ausencia de dolor); 10 (peor dolor padecido/imaginable). } \\
\hline Día & 0 & 7 & 14 & 21 & 28 & 35 & 42 & 49 & 56 & 63 & 70 & Cont. \\
\hline EVA & & & & & & & & & & & & \\
\hline 10 & & & & & & & & & & & & \\
\hline 9 & $x$ & & & & & & & & & & & \\
\hline 8 & & & & & & & & & & & & \\
\hline 7 & & & & & & & & & & & & \\
\hline 6 & & $x$ & $x$ & & & & & & & & & \\
\hline 5 & & & & $x$ & & & & & & & & \\
\hline 4 & & & & & & & & & & & & \\
\hline 3 & & & & & $x$ & $x$ & $x$ & & & & & \\
\hline 2 & & & & & & & & $x$ & $x$ & & & \\
\hline 1 & & & & & & & & & & $x$ & $x$ & \\
\hline 0 &
\end{tabular}

\begin{tabular}{|l|l|l|l|l|l|l|l|l|l|l|l|l|}
\hline Limitaciones. & 0 & 7 & 14 & 21 & 28 & 35 & 42 & 49 & 56 & 63 & 70 & Cont. \\
\hline Día & 1 & 1 & 1 & 1 & 0 & 0 & 0 & 0 & 0 & 0 & 0 & \\
\hline Caminar & 2 & 2 & 2 & 1 & 1 & 1 & 0 & 0 & 0 & 0 & 0 & \\
\hline Curación & 1 & 1 & 1 & 1 & 1 & 1 & 1 & 0 & 0 & 0 & 0 & \\
\hline Vestirse & 3 & 3 & 2 & 2 & 2 & 1 & 1 & 1 & 0 & 0 & 0 & \\
\hline Dormir & 3 & 2 & 2 & 1 & 1 & 1 & 1 & 0 & 0 & 0 & 0 & \\
\hline Trabajo & 3 & 3 & 2 & 2 & 2 & 1 & 1 & 0 & 0 & 0 & 0 & \\
\hline Alodinia & &
\end{tabular}

Puntuación. 0: no limitación. 1: puede con mínima limitación. 2: Puede con dificultad. 3: no puede. (Se agregó la alodinia como marcador en el score por responsabilidad en las limitaciones)

\begin{tabular}{|l|l|l|l|l|l|l|l|l|l|l|l|l|}
\hline Evolución de la lesión (trofismo tisular). \\
\hline Día & 0 & 7 & 14 & 21 & 28 & 35 & 42 & 49 & 56 & 63 & 70 & Cont. \\
\hline Tamaño & & & & & & & & & & & & \\
\hline Borde & & & & & & & & & & & & \\
\hline Lecho & & & & & & & & & & & & \\
\hline $\begin{array}{l}\text { Piel } \\
\text { perilesional }\end{array}$ & & & & & & & & & & & & \\
\hline
\end{tabular}

Puntuación. 0: favorable. 1: sin variantes. 2: desfavorable

responder favorablemente, mientras que los pacientes portadores de lesiones de origen vasculítico por afecciones reumáticas respondieron favorablemente en menor período de tiempo.

Muchos de los pacientes mejoraron las aptitudes de la calidad de vida, favoreciendo la recuperación y calidad del sueño, la recuperación de actividades cotidianas, una modificación evidente de la timia y un número significativo disminuyó la ingesta de medicación opioide, quedando con medicación analgésica como los antiinflamatorios no esteroides.

Con respecto a la acción trófica del procedimiento, se comprobó la mejoría de la cicatrización para las heridas crónicas refractarias, que a partir de las infiltraciones experimentaron una modificación del lecho en mayor o menor grado con favorecimiento del tejido de granulación, mejoramiento de la piel perilesional, el borde de la herida y la reducción del tamaño de acuerdo al seguimiento tabulario utilizado.

Se pudieron realizar procedimientos que antes del inicio del tratamiento no eran viables, como la realización

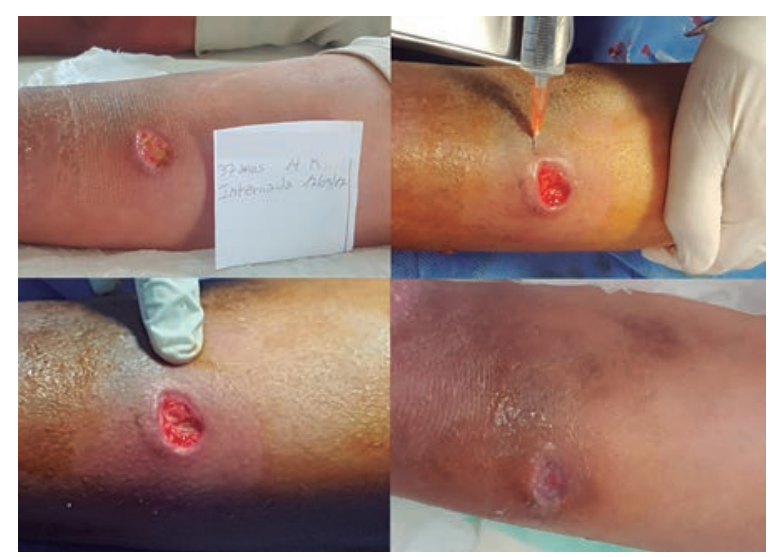

Figura 7.

de injertos de piel en 18 de los pacientes a partir de los 3 meses del inicio, con resultados favorables.

Además se comprobó una mejoría de las lesiones en miembros inferiores en la mayoría de los pacientes (más del 80\%) y en un 30\% el cierre de las heridas, asociado a curas habituales en el Servicio de Cirugía Plástica (PHMB, propóleos gasa o loción $)^{21}$.

Se agrega como dato de aporte, además del tratamiento del dolor crónico, el hecho de favorecer la zona receptora de injertos de piel en lámina o pinch grafting para pacientes con lesiones ulcerosas vasculíticas y diabéticas refractarias.

Se infiere, atento a la evolución, que la infiltración perineural de dextrosa podría haber influido clínicamente en la carga bacteriana de las heridas en la mayoría de los casos, dado que la mejoría y el favorecimiento de los procesos de reparación no hubieran sido posibles de no haber sido de esa forma. Los cultivos tomados el día 0 y los siguientes resultados de 30/45 días demostraron una importante reducción en la presencia de gérmenes, siendo los más hallados el Staphylococus aureus coagulasa negativo y la Pseudomona aeruginosa.

Durante el seguimiento de los pacientes no hubo modificaciones significativas en la glucemia mediante Hemoglucotest (HGT) en la mayoría de los pacientes.

Durante el desarrollo del presente trabajo se observaron los siguientes efectos adversos: ardor intraaplicación (tolerable, que no impidió procedimiento) en el $15,7 \%$ (8 pacientes) y celulitis de miembro inferior en el 7,8\% (4 pacientes).

\section{CASO 1 (Figura 7)}

- Femenina, 37 años.

- Diagnóstico: artritis reumatoidea, úlcera de 6 meses de evolución.

Infiltración subcutánea perineural + cura húmeda. Cierre: 25 días. Día 0: EVA 9, día 15: EVA 0.

\section{CASO 2 (Figura 8)}

- Masculino 70 años.

- Diagnóstico: pioderma gangrenoso. 


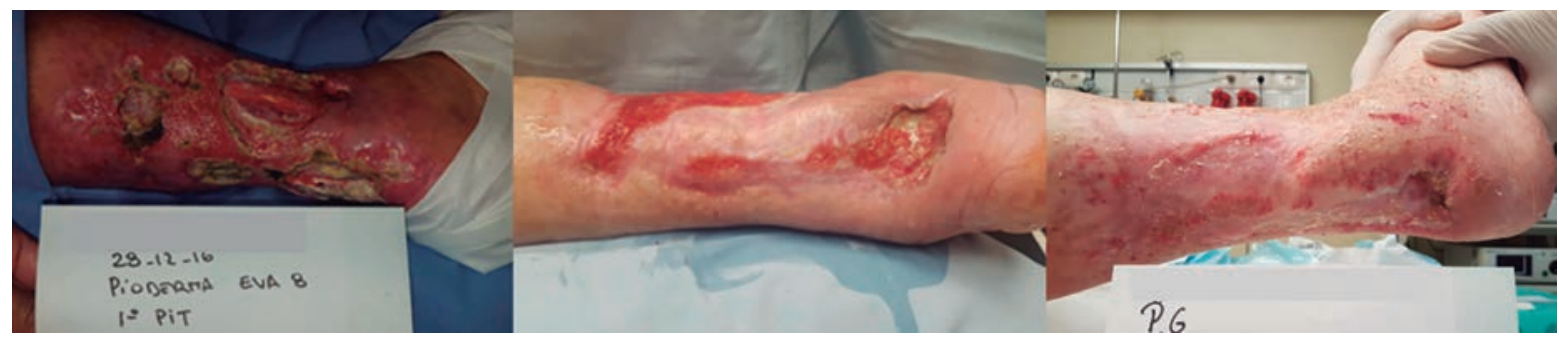

Figura 8.

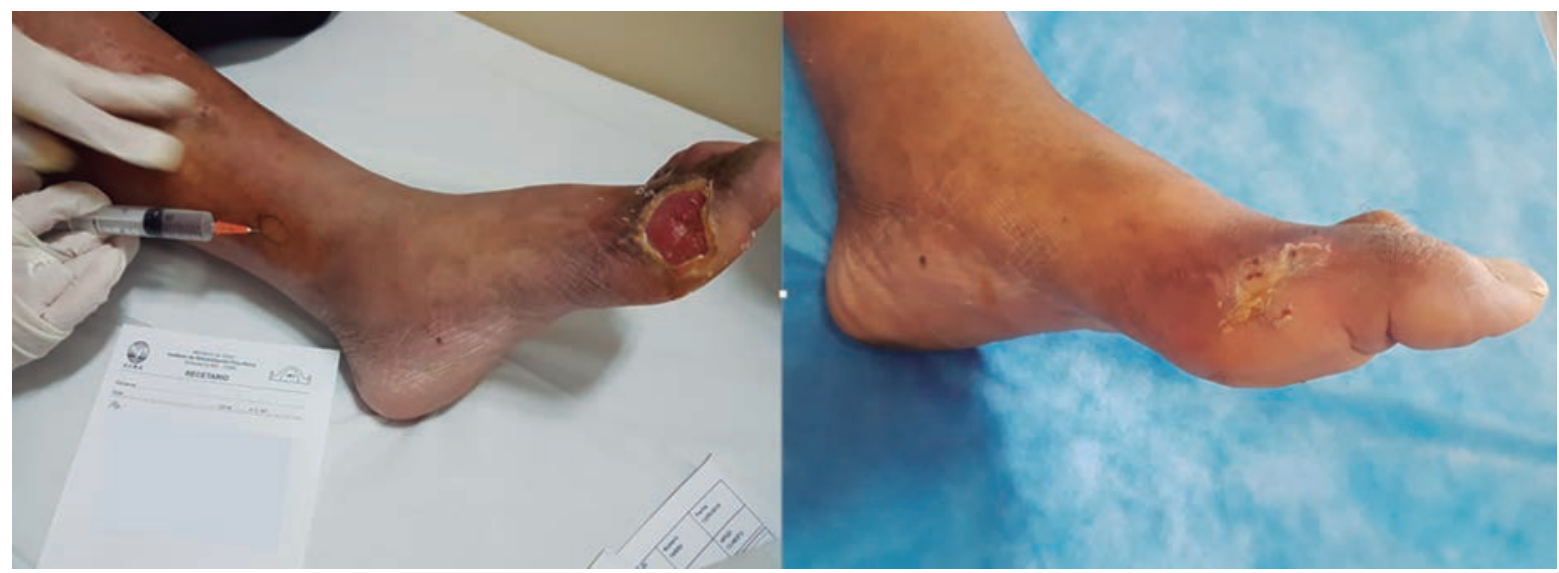

Figura 9.

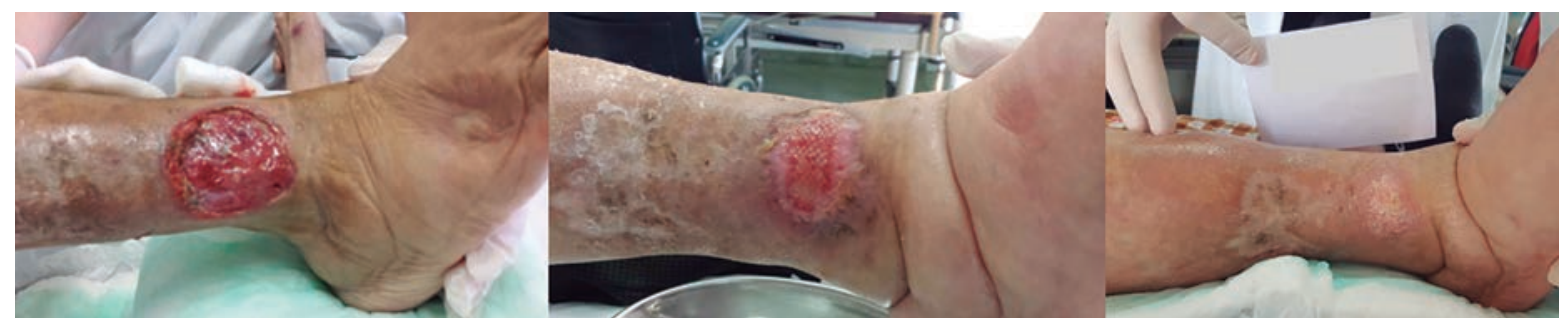

Figura 10.

- Infiltración subcutánea perineural + cura húmeda oclusiva. Cierre: 120 días. Día 0: EVA 8, día 15: EVA 2, día 25: EVA 0.

\section{CASO 3 (Figura 9)}

- Masculino 61 años.

- Diagnóstico: pie diabético.

- Infiltración subcutánea perineural+ gasa con propóleos. Cierre 35 días. Día 0: EVA 5, día 15: EVA 0.

\section{CASO 4 (Figura 10)}

- Femenino 64 años.

- Diagnóstico: artritis reumatoidea.

- Infiltración subcutánea perineural + gasa con propóleos. Cierre 50 días. Día 0: EVA 6, día 15: EVA 1.

\section{DISCUSIÓN}

La presencia de dolor en heridas complejas de diferentes etiologías es una de las barreras a tratar para facilitar la resolución del daño tisular, comprendiendo que para el paciente es tan importante el dolor como la herida.
El DC es un dolor de difícil localización y tiene diferentes formas de presentación (descarga, quemazón, sordo), cuya evolución no guarda relación con la evolución de la herida ni el grado de daño tisular.

Se manifiesta como una hiperalgesia o alodinia (percepción anormal del dolor en situaciones que no deberían producirlo) tendiendo a ser cuantitativamente superior.

A través del desarrollo del trabajo y evaluación de los resultados se trata de demostrar la utilidad y efectividad del procedimiento de infiltración perineural subcutánea de una solución en base a dextrosa al 5\% para el manejo del DC en úlceras de miembro inferior y cicatrización en heridas crónicas.

También, en asociación con los resultados obtenidos, se evaluaron la tolerancia y el sostenimiento del tratamiento, y además la disponibilidad de los elementos utilizados y el tipo de relación costo/beneficio.

\section{LIMITACIONES}

Nuestro estudio explora la eficacia del uso de la infiltración subcutánea perineural para el manejo del DC en he- 
ridas complejas de diversas etiologías. Debido al carácter innovador de este trabajo, es necesario continuar con nuevos estudios comparativos, con un período mayor de seguimiento, ampliando además el estudio en la reparación tisular y el efecto de la infiltración sobre la carga bacteriana. Dadas las características del DC, se deberá evaluar la repercusión del procedimiento y su condicionamiento psíquico como motivador de la respuesta individual.

\section{CONCLUSIÓN}

La técnica descrita por el Dr. John Lyftogt en DC asociado a heridas complejas de miembro inferior es un procedimiento nuevo, inédito, seguro y económico, que ha demostrado su efectividad como coadyuvante de esta compleja entidad, favoreciendo además la cicatrización de los tejidos afectados.

\section{BIBLIOGRAFÍA}

1. Ballantyne JC, BhatnagarS, Blyth F, et al. IASP statement on opioids. Washington, DC: International Association for the Study of Pain, February 2018 (http://www.iasppain.org/Advocacy/Content.aspx?/temNumber=7194).

2. Edwards RR, Dworkin RH, Sullivan MD, Turk DC, Wasan AD. The role ofpsychosocialprocesses in thedevelopment and maintenance of chronic pain. J Pain 2016;17 Suppl:T70-T92.

3. Finnerup NB. Nonnarcotic methods of pain management. N Engl J Med. 2019;380(25):2440-2448.

4. Mick G, Baron R, Finnerup NB, et al. What is localized neuropathic pain? A first proposal to characterize and define a widely used term. Pain Manage 2012;2:71-7.

5. Ballantyne JC, Bhatnagar S, Blyth F, et al. IASP statement on opioids. Washington, DC: International Associationforthe Study of Pain, February 2018 (http://www.iasp-pain.org/Advocacy/Content.aspx?ltemNumber $=7194$

6. Agentes tópicos o apósitos para el dolor en las úlceras venosas de la pierna. Cochrane Systematic Review - Intervention Version published: 20 January 2003. See what's newhttps://doi. org/10.1002/14651858.CD001177.

7. Wall EHEMVD. Capsaicin-sensitive nerves and energy homeostasis: involvement in satiety and s.n. University of Groningen, NL. 2005. 192 p.

8. Bastami S, Frödin T, Ahlner J, Uppugunduri S. Topical morphine gel in the treatment of leg ulcers, a double-blind, placebo-controlled clinical trial: a pilot sutdy. Int Wound J 2012 (4:419-27).

9. Zeppetella G, Paul J, Ribeiro MD, Analgesic Efficacy of Morphine Applied Topically to Painful Ulcer. Journal of Pain and Symptom Management 2003;25(6):555-8.

10. A Systematic Review of Dextrose Prolotherapy for Chronic Musculoskeletal Pain https://www.ncbi.nlm.nih.gov/pmc/articles/ PMC 4938120/.
11. Distel LM, Best TM. Prolotherapy: a clinical review of its role in treating chronic musculoskeletal pain. PM R. 2011; 3 (6 Suppl 1): S78-S81.)

12. Bernard C. Eds. Baillere et Fils. Paris: 1854. Lecons de physiologie experimentale appliqués a la medecine.

13. Hackett GS, Hemwall GA, Montgomery GA. Ligament and tendon relaxation treated by prolotherapy. 5th edition. Oak Park (IL): Gustav A. Hemwall; 1993.

14. Lyftogt J. Prolotherapy and Achilles tendinopathy: A prospec-tive pilot study of an old treatment. Australas Musculoskeletal Med 2005;10(1):16-9.

15. Morales-Lázaro S, Simon SA, Rosenbaum T. The role of endogenous molecules in modulating pain through transient receptor potential vanilloid 1 (TRPV1). J Physiol 2013;591(13):3109-21.

16. Bertrand H, Kyriazis M, Dean Reeves K, Lyftogt J, Rabago D. El manitol tópico reduce el dolor inducido por la capsaicina: resultados de un ensayo piloto aleatorizado, doble ciego y controlado. PM R 12 de mayo de 2015.

17. Lyftogt J. Subcutaneous prolotherapy treatment of refractory knee, shoulder and lateral elbow pain. Australia's Musculoskeletal Medicine Journal 2007:2:10-112.

18. Kessner SS, Bingel U, Thomalla G. Somatosensory deficits after stroke: a scoping review. Top StrokeRehabil 2016:23(2):136-46.

19. Hilton J. The Classic: On Rest and Pain: Lecture XIV. Clin Orthop Relat Res 2009;467(9):2208-14.

20. Watson P. The recovergram. Australas Musculoskeletal Med 2000;5(2):24-8

21. Leoni HT, Amoroso A, Aponte P, Ross N, Olivero Vila F. Utilización de propóleos en heridas complejas. Revista Argentina de Cirugía Plástica 2017;(2):0065-0071. 